British Journal of Mathematics \& Computer Science 20(4): 1-10, 2017; Article no.BJMCS.31342

ISSN : 2231-0851

SCIENCEDOMAIN international www.sciencedomain.org

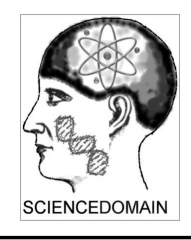

\title{
Existence and Uniqueness of Positive Periodic Solution of an Extended Rosenzweig-MacArthur Model via Brouwer's Topological Degree
}

\author{
Enobong E. Joshua ${ }^{1}$, Ekemini T. Akpan ${ }^{2}$, Olukayode Adebimpe ${ }^{3}$ \\ and Chinwendu E. Madubueze ${ }^{4}$ \\ ${ }^{1}$ Department of Mathematics/Statistics, University of Uyo, Uyo, Nigeria. \\ ${ }^{2}$ Department of Science Education, University of Uyo, Uyo, Nigeria. \\ ${ }^{3}$ Department of Physical Sciences, Landmark University, Omu-Aran, Nigeria. \\ ${ }^{4}$ Department of Mathematics/Statistics/Computer Science, University of Agriculture, Makurdi,
}

Nigeria.

Authors' contributions

This work was carried out in collaboration between all authors. Author EEJ formulated a dimensionless dynamical system and generated its numerical simulations, author ETA used Brouwer's and coincidence degree algorithm to established existence of solutions. Authors OA and CEM proved the uniqueness of the periodic solution. The final manuscript was completed by author ETA. All authors read and approved the final manuscript.

Article Information

DOI: $10.9734 /$ BJMCS/2017/31342

Editor(s):

(1) Zhenkun Huang, School of Science, Jimei University, P. R. China.

Reviewers:

(1) Abdullah Sonmezoglu, Bozok University, Turkey.

(2) Hui Zhang, Xian Research Institute of High-tech Hongqing Town, China.

Complete Peer review History: http://www.sciencedomain.org/review-history/17772

Original Research Article

Received: $31^{\text {st }}$ December 2016

Accepted: $20^{\text {th }}$ January 2017

Published: $9^{\text {th }}$ February 2017

\begin{abstract}
The necessary conditions for existence of periodic solutions of an Extended RosenzweigMacArthur model are obtained using Brouwer's degree. The forward invariant set is formulated to ensure the boundedness of the solutions, using Brouwers fixed point properties, and Zorns lemma. Also, sufficient conditions for the existence of a unique positive periodic solution have been established using Barbalats lemma and Lyapunovs functional. Numerical responses show that, the phase-flows of the non-autonomous system exhibit an asymptotically stable periodic solution which is globally attractive and trapped in the absorbing region.
\end{abstract}

*Corresponding author: E-mail: ekeminitakpan@uniuyo.edu.ng; 
Keywords: Periodic solutions; global attractivity; brouwers degree; lyapunovs functional.

2010 Mathematics Subject Classification: 53C25, 83C05, 57N16.

\section{Introduction}

Mathematical modelling of ecological system has explored robust modifications in terms of the nature of their interactions (i.e., competitive, prey-predator systems, spatio-temporal dynamics, coope- rative systems, patch-diffusion, delay systems and so on), functional responses (i.e., Holling types, Leslie-Gower, Beddington-DeAngelis, and so on) and ecologically perturbative parameters. In prey-predator systems, it is pertinent to assume that all biological and environmental perturbative parameters and state variables are subject to natural fluctuations in time. Thus, the assumption of periodically varying perturbative parameters is a way of making the dynamical system more realistic as compared to constant perturbative parameters. Obviously, periodic variations in the environment and ecologically perturbative parameters are characterized by seasonal effect of weather, food supplies, predation effects, mating durations, time delay due to gestation, and so on.

The qualitative dynamical behaviors of these mathematical models are widely studied in populations of multiple interacting species in the ecosystem. [1] investigated the existence and global attractivity of positive periodic solutions for a Holling II two-prey and one-predator system. Periodic solutions for a three-species Lotka-Volterra food chain model with time delay were studied in [2]. They derived the sufficient conditions for the existence of positive periodic solutions of the system. In the same [3], obtained the necessary and sufficient conditions for existence of periodic solutions of predator-prey dynamical system with Beddington-DeAngelis-type functional response. Existence of periodic solutions for a two-species non-autonomous competitive Lotka-Volterra patch system with time delay was established in [4].

Exploration of these robust dynamical systems requires using topological degree theory, see [5] [6] [7]. In this theory, to prove the existence of solution for a natural abstract formulated IVP, say

$$
\left\{\begin{array}{l}
\dddot{X}=F(t, \ddot{X}(t), \dot{X}, X(t) ; t \in[0, \omega] \\
F \subset C^{1}:[0, \omega] \times \mathbb{R}^{3} \rightarrow \mathbb{R}^{3} \\
X(0)=X(\omega), \dot{X}(0)=\dot{X}(\omega), \ddot{X}(0)=\ddot{X}(\omega), X(t)=X(t+\omega)
\end{array}\right.
$$

usually reduces to solving the abstract operator equation, $L(X)=N(X)$ which has some topological degree properties, see [8]. Moreover, results of theorems, and propositions well established via Topological Degree Theory can be numerically simulated using sophisticated dynamical tools (e.g MAPLE) [9] [10].

\section{Model Formulation and Its Invariance Region}

The Extended Rosenzwieg-MacArthur Model formulated and studied in [11] is given as:

$$
\left\{\begin{array}{l}
\frac{d x_{1}}{d t}=r x_{1}-\frac{r x_{1}^{2}}{K}-a_{2} \frac{x_{1}}{b_{1}+x_{1}} x_{2}-a_{3} \frac{x_{1}}{b_{1}+x_{1}} x_{3} \\
\frac{d x_{2}}{d t}=c_{2} a_{2} \frac{x_{2}}{b_{1}+x_{1}} x_{3}-d_{2} x_{2}-a_{3} \frac{x_{2}}{b_{2}+x_{2}} x_{3} \\
\frac{d x_{3}}{d t}=c_{3} a_{3} \frac{x_{2}}{b_{2}+x_{2}} x_{3}-d_{3} x_{3}+c_{3} a_{3} \frac{x_{1}}{b_{1}+x_{1}} x_{3}
\end{array}\right.
$$


where $x_{1}(t), x_{2}(t)$, and $x_{3}(t)$ are the population densities of the interacting species and $r, K, a_{2}, a_{3}, b_{1}$, $b_{2}, c_{2}, c_{3}, d_{2}$ and $d_{3}$ are positive ecological parameters. In [12] a topologically equivalent dynamical model of system (2.1) was obtained via non-dimensionalization of the state variables as follows:

$$
\left\{\begin{array}{l}
\frac{d x}{d \tau}=\alpha u-\frac{\alpha u^{2}}{\kappa}-\eta \frac{u}{1+u} v-\frac{u}{1+u} w \\
\frac{d y}{d \tau}=\varepsilon \frac{u}{1+u} v-\xi v-\sigma \frac{v}{1+v} w \\
\frac{d z}{d \tau}=\beta \frac{v}{1+v} w-\mu w+\beta \frac{u}{1+u} w
\end{array}\right.
$$

where $x(\tau)=\frac{x_{1}(t)}{b_{1}}, y(\tau)=\frac{x_{2}(t)}{b_{2}}, z(\tau)=\frac{x_{3}(t)}{b_{1}}, \alpha=\frac{r}{a_{3}}, \kappa=\frac{K}{b_{1}}, \eta=\frac{a_{2} b_{2}}{a_{3} b_{1}}, \varepsilon=\frac{c_{2} a_{2}}{a_{3}}, \xi=\frac{d_{2}}{a_{3}}, \sigma=$ $\frac{b_{1}}{b_{2}}, \mu=\frac{d_{3}}{a_{3}}, \tau=a_{3} t, c_{3}=\beta$. Suppose the ecological parameters are periodic functions, so system $(2.2)$ can be modified to a non-autonomous system as follows:

$$
\left\{\begin{array}{l}
\frac{d u}{d \tau}=\alpha(\tau)-\frac{\alpha(\tau) \exp u(\tau)}{\kappa(\tau)}-\eta(\tau) \frac{\exp v(\tau)}{1+\exp u(\tau)}-\frac{\exp w(\tau)}{1+\exp u(\tau)} \\
\frac{d v}{d \tau}=\varepsilon(\tau) \frac{\exp u(\tau)}{1+\exp u(\tau)}-\xi(\tau)-\sigma(\tau) \frac{\exp w(\tau)}{1+\exp v(\tau)} \\
\frac{d w}{d \tau}=\beta(\tau) \frac{\exp v(\tau)}{1+\exp v(\tau)}-\mu(\tau)+\beta(\tau) \frac{\exp u(\tau)}{1+\exp u(\tau)}
\end{array}\right.
$$

where $u(\tau)=\operatorname{In}|x(\tau)|, v(\tau)=$ In $|y(\tau)|, w(\tau)=\operatorname{In}|z(\tau)|, \alpha(\tau)=\alpha(\tau+\omega), \eta(\tau)=$ $\eta(\tau+\omega), \varepsilon(\tau)=\varepsilon(\tau+\omega), \xi(\tau)=\xi(\tau+\omega), \sigma(\tau)=\sigma(\tau+\omega), \beta(\tau)=\beta(\tau+\omega), \mu(\tau)=\mu(\tau+\omega)$, and subject to initial conditions, $u(0)=u_{0}>0, v(0)=v_{0}>0, w(0)=w_{0}>0$.

Using the fundamental theorem of calculus, it is easy to see that $\mathbb{R}_{+}^{3}$ is the invariance region of solutions of system (2.3) satisfying;

$$
\left\{\begin{array}{l}
u(\tau)=u_{o} \exp \int_{0}^{\omega}\left\{\alpha(\tau)-\frac{\alpha(\tau) \exp u(\tau)}{\kappa(\tau)}-\eta(\tau) \frac{\exp v(\tau)}{1+\exp u(\tau)}-\frac{\exp w(\tau)}{1+\exp u(\tau)}\right\} d s \\
v(\tau)=v_{0} \exp \int_{0}^{\omega}\left\{\varepsilon(\tau) \frac{\exp u(\tau)}{1+\exp u(\tau)}-\xi(\tau)-\sigma(\tau) \frac{\exp w(\tau)}{1+\exp v(\tau)}\right\} d s \\
w(\tau)=w_{0} \exp \int_{0}^{\omega}\left\{\beta(\tau) \frac{\exp v(\tau)}{1+\exp v(\tau)}-\mu(\tau)+\beta(\tau) \frac{\exp u(\tau)}{1+\exp u(\tau)}\right\} d s
\end{array}\right.
$$

Thus, the state variables are invariants in the positive octant cone, $\mathbb{R}_{+}^{3}=\left(((u \tau), v(\tau), w(\tau))^{T} \in\right.$ $\left.\mathbb{R}^{3}: u(\tau)>0, v(\tau)>0, w(\tau)>0\right)$.

\section{Some Results on Brouwer's Topological Degree Theory}

\subsection{Lemma $1[13]$}

Assume $f: \mathbb{T} \subset \mathbb{R} \rightarrow \mathbb{R}$ is $\omega$-periodic function, let $\tau_{1}, \tau_{1} \in[0, \omega]$ then, $\bar{f}=\frac{1}{\omega} \int_{0}^{\omega}|f(\tau)| d(\tau), f^{l}=$ $\operatorname{minf}(\tau), f^{m}=\max f(\tau)$ 
$\forall \tau \in[0, \omega]$ and

$$
\left\{\begin{array}{l}
f(\tau) \leq f\left(\tau_{1}\right)+\int_{0}^{\omega}|\dot{f}(s)| d s \\
f(\tau) \geq f\left(\tau_{2}\right)-\int_{0}^{\omega}|\dot{f}(s)| d s
\end{array}\right.
$$

\section{$3.2 \quad$ Lemma $2[8]$}

Let $X$ and $Y$ be two Banach spaces and let $L: D o m L \subset X \rightarrow Y$ be a linear operator. Let $N: X \rightarrow Y$ be a continuous mapping. A mapping $F: D o m L \subset X \rightarrow Y$ is said to be a Fredholm mapping of index zero, if $\operatorname{dimKer} L=\operatorname{codim} \operatorname{Im} L<\infty$ and $\operatorname{Im} L$ is closed in $Y$. If $L$ is a Fredholm mapping, its index is an integer $\operatorname{Ind} L=\operatorname{dim} L-\operatorname{codim} \operatorname{Im} L$. Suppose $L$ is a Fredholm mapping of index zero, there exist continuous projections, $P: X \rightarrow X$ and $Q: Y \rightarrow Y$ such that $\operatorname{Im} P=$ $\operatorname{Ker} L, \operatorname{Im} L=\operatorname{Ker} Q=\operatorname{Im}(I-Q)$, and the restriction $L_{P}$ of $L$ to $\operatorname{Dom} L \cap K \operatorname{KerP}:(I-Q) X \rightarrow \operatorname{ImL}$ is invertible. Denote the generalized inverse of $L_{P}$ by $K_{P}$ such that $L K_{P}=I$, and $K_{P} L=I-P$. Let $\Omega$ be a non-empty, open bounded subset of $X$, then the mapping $N$ is said to be $L$-compact on $\bar{\Omega}$ if the mapping $Q N: \Omega \rightarrow Y$ is continuous, $Q N(\bar{\Omega})$ is bounded, and $K_{P}(I-Q) N: \bar{\Omega} \rightarrow X$ is compact (i.e., it is continuous, and $K_{P}(I-Q) N(\bar{\Omega})$ relatively compact). Since $\operatorname{Im} Q$ is isomorphic to $\operatorname{Ker} L$, then there exists an isomorphism $J: \operatorname{Im} Q \rightarrow \operatorname{Ker} L$.

\section{$3.3 \quad$ Lemma $3[7]$}

Let $\Omega \in \mathbb{R}^{n}$ be an open bounded set and $L: \bar{\Omega} \rightarrow R^{n}$ be a continuous mapping. If $p \notin L(\partial \Omega)$, then the Brouwer degree of $L$ at $p$ relative to $\Omega$ is an integer number, denoted by: $\operatorname{deg}(L, \Omega, p)=$ sign $\left|J_{p}(p)\right|$, where $J_{P}(p)$ is the Jacobian matrix of the operator $L$ at $p$, satisfying the following properties:

i $\operatorname{deg}(I, \Omega, p)=1$, iff $p \in \Omega$, where $I$ denotes the identity mapping.

ii if $\operatorname{deg}(L, \Omega, p) \neq 0$ then $L x=p$ has a solution in $\Omega$

iii if $H(t, x):[0,1] \times \bar{\Omega} \rightarrow R^{n}$ is a continuous homotopic mapping defined as $H(t, x)=t \phi(x)+$ $(1-t) \psi(x)$ for $\phi, \psi \in C^{1}(\Omega)$, and $\forall p \in \mathbb{R}^{n} \backslash H(t, \partial \Omega)$, then $\operatorname{deg}(\phi, \Omega, p)=\operatorname{deg}(\psi, \Omega, p)$ and $\operatorname{deg}(H(t, x), \Omega, p)=\operatorname{deg}(H(0, x), \Omega, p)$ independent of $t \in[0,1]$.

\subsection{Lemma $4[5]$}

Let $\Omega$ be an open bounded set. Let $L$ be a Fredholm mapping of index zero, and $N$ be $L-$ compact on $\bar{\Omega}$. Assume

i for each $t \in(0,1)$ every solution $x$ of $L x=t N x$, is such that $x \notin D o m L \cap \partial \Omega$.

ii $Q N x \neq 0, \forall x \in D o m L \cap \partial \Omega$, and

iii $\operatorname{deg}(J Q N: \operatorname{Ker} L \cap \Omega, 0) \neq 0$ where $J: \operatorname{Im} Q \rightarrow \operatorname{Ker} L$ is an Isomorphism and $\operatorname{deg}$ denotes the Brouwer topological degree.

Then the operator equation, $L x=N x$ has at least one solution in $D o m L \cap \partial \Omega$.

\section{Existence of Positive Periodic Solutions}

\subsection{Proposition 1}

Assuming that the perturbation parameters of system (2.3) are periodic functions, then system (2.3) has at least one positive periodic solution. 
Proof: Suppose $X=Y=(u(\tau), v(\tau), w(\tau))^{T} \in C_{c}^{1}\left(\mathbb{R}, \mathbb{R}^{3}\right): u(\tau)=u(\tau+\omega), v(\tau)=v(\tau+$ $\omega), w(\tau)=w(\tau+\omega)$ is the phase flows system (2.3), then equipped the spaces, $X$ and $Y$ with the usual Euclidean norm, say $\|u(\tau), v(\tau), w(\tau)\|=\max |u(\tau)|+\max |v(\tau)|+\max |w(\tau)| \forall \tau \in$ $[0, \omega]$. Denote $L: D o m L \subset X \rightarrow Y$ and $N: X \rightarrow Y$ as operator equations,

$$
\begin{gathered}
L(u(\tau), v(\tau), w(\tau))^{T}=(\dot{u}(\tau), \dot{v}(\tau), \dot{w}(\tau)) \\
N(u(\tau), v(\tau), w(\tau))^{T}=\left\{\begin{array}{l}
\alpha(\tau)-\frac{\alpha(\tau) \exp u(\tau)}{\kappa(\tau)}-\eta(\tau) \frac{\exp v(\tau)}{1+\exp u(\tau)}-\frac{\exp w(\tau)}{1+\exp u(\tau)} \\
\varepsilon(\tau) \frac{\exp u(\tau)}{1+\exp u(\tau)}-\xi(\tau)-\sigma(\tau) \frac{\exp w(\tau)}{1+\exp v(\tau)} \\
\beta(\tau) \frac{\exp v(\tau)}{1+\exp v(\tau)}-\mu(\tau)+\beta(\tau) \frac{\exp u(\tau)}{1+\exp u(\tau)}
\end{array}\right.
\end{gathered}
$$

Define two continuous projectors $P: X \rightarrow X$ and $Q: Y \rightarrow Y$ as

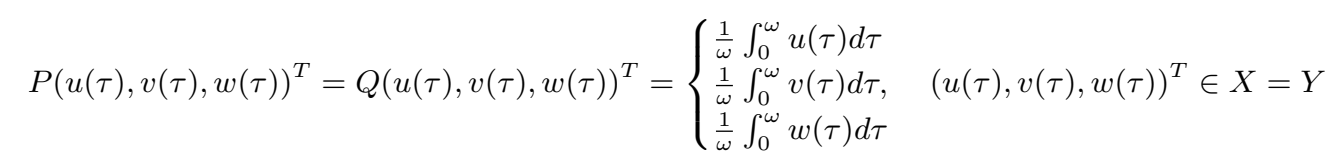

It is clear that $\operatorname{Ker} L=\left(\mathbf{x} \in X: \mathbf{x}=\mathbf{h}, \mathbf{h} \in \mathbb{R}^{3}\right)$, and $\operatorname{Im} L=\left(\mathbf{y} \in Y: \int_{0}^{\omega} \mathbf{y}(\tau) d \tau=0\right)$ is closed in $Y$. Observe that $\operatorname{dim} \operatorname{Ker} L=$ codim $\operatorname{Im} L=3, \operatorname{ImP}=\operatorname{Ker} L, \operatorname{Ker} Q=\operatorname{Im} L Q=\operatorname{Im}(I-Q)$ Therefore, $L$ is a Fredholm mapping of index zero.

Furthermore, the generalized inverse $K_{P}$ of $L_{P}$ has the form $K_{P}: \operatorname{ImL} \rightarrow \operatorname{DomL} \cap \operatorname{Ker} P$,

$$
K_{p} y=\int_{0}^{\tau} y(s) d s-\frac{1}{\omega} \int_{0}^{\omega} \int_{0}^{\tau} y(s) d s d \tau
$$

Then, $Q N: X \rightarrow Y$ yields

$$
Q N \mathbf{x}=\left\{\begin{array}{l}
\frac{1}{\omega} \int_{0}^{\omega}\left(\alpha(\tau)-\frac{\alpha(\tau) \exp u(\tau)}{\kappa(\tau)}-\eta(\tau) \frac{\exp v(\tau)}{1+\exp u(\tau)}-\frac{\exp w(\tau)}{1+\exp u(\tau)}\right) d \tau \\
\frac{1}{\omega} \int_{0}^{\omega}\left(\varepsilon(\tau) \frac{\exp u(\tau)}{1+\exp u(\tau)}-\xi(\tau)-\sigma(\tau) \frac{\exp w(\tau)}{1+\exp v(\tau)}\right) d \tau \\
\frac{1}{\omega} \int_{0}^{\omega}\left(\beta(\tau) \frac{\exp v(\tau)}{1+\exp v(\tau)}-\mu(\tau)+\beta(\tau) \frac{\exp u(\tau)}{1+\exp u(\tau)}\right) d \tau
\end{array}\right.
$$

and $K_{p}(I-Q) N: X \rightarrow X$ yields

$$
K_{p}(I-Q) N \mathbf{x}=\int_{0}^{\tau} N \mathbf{x} d s-\frac{1}{\omega} \int_{0}^{\omega} \int_{0}^{\tau} N \mathbf{x} d s d \tau-\frac{1}{\omega} \int_{0}^{\tau} \int_{0}^{\omega} N \mathbf{x} d s d s+\frac{1}{\omega^{2}} \int_{0}^{\omega} \int_{0}^{\tau} \int_{0}^{\omega} N \mathbf{x} d s d s d \tau
$$

Clearly, by Lebesgue convergence theorem, $Q N$ and $K_{P}(I-Q) N$ are continuous maps. Since the maps are well-defined on finite dimensional Banach spaces, by Arzela-Ascoli theorem, $K_{P}(I-$ $Q) N(\bar{\Omega})$ is relatively compact. Additionally, $Q N(\bar{\Omega})$ is bounded for any open bounded set $\Omega \subset X$, and $N$ is $L$ - compact.

We now seek a forward invariance set $K \subset X$ that is convex and compact such that the phase flows $\Phi(\tau) \subset K$ satisfy the operator equation $L x=t N x, t \in(0,1)$. Consider

$$
\left\{\begin{array}{l}
\dot{u}(\tau)=\alpha(\tau)-\frac{\alpha(\tau) \exp u(\tau)}{\kappa(\tau)}-\eta(\tau) \frac{\exp v(\tau)}{1+\exp u(\tau)}-\frac{\exp w(\tau)}{1+\exp u(\tau)} \\
\dot{v}(\tau)=\varepsilon(\tau) \frac{\exp u(\tau)}{1+\exp u(\tau)}-\xi(\tau)-\sigma(\tau) \frac{\exp w(\tau)}{1+\exp v(\tau)} \\
\dot{w}(\tau)=\beta(\tau) \frac{\exp v(\tau)}{1+\exp v(\tau)}-\mu(\tau)+\beta(\tau) \frac{\exp u(\tau)}{1+\exp u(\tau)}
\end{array}\right.
$$


integrating yields

$$
\left\{\begin{array}{l}
\omega \bar{\alpha}=\int_{0}^{\omega}\left(\alpha(\tau)-\frac{\alpha(\tau) \exp u(\tau)}{\kappa(\tau)}-\eta(\tau) \frac{\exp v(\tau)}{1+\exp u(\tau)}-\frac{\exp w(\tau)}{1+\exp u(\tau)}\right) d \tau \\
\omega \bar{\xi}=\int_{0}^{\tau}\left(\varepsilon(\tau) \frac{\exp u(\tau)}{1+\exp u(\tau)}-\xi(\tau)-\sigma(\tau) \frac{\exp w(\tau)}{1+\exp v(\tau)}\right) d \tau \\
\omega \bar{\mu}=\int_{0}^{\omega}\left(\beta(\tau) \frac{\exp v(\tau)}{1+\exp v(\tau)}-\mu(\tau)+\beta(\tau) \frac{\exp u(\tau)}{1+\exp u(\tau)}\right) d \tau
\end{array}\right.
$$

and

$$
\left\{\begin{array}{l}
\int_{0}^{\omega}|\dot{u}(\tau)| d \tau \leq \int_{0}^{\omega}(\alpha(\tau)+|\alpha(\tau)|) d \tau=\omega \overline{(\alpha+|\alpha|)} \\
\left.\int_{0}^{\omega}|\dot{v}(\tau)| d \tau \leq \int_{0}^{\omega} \xi(\tau)+|\xi(\tau)| d \tau\right)=\omega \overline{(\xi+|\xi|)} \\
\left.\int_{0}^{\omega}|\dot{w}(\tau)| d \mu \leq \int_{0}^{\omega} \mu(\tau)+|\mu(\tau)| d \tau\right)=\overline{\omega(\mu+|\mu|)}
\end{array}\right.
$$

Using Mean-Value Theorem for integral equations, we have that there exists $\delta_{i} \in[0, \omega]$ for $i=1,2,3$ such that $u\left(\delta_{1}\right) \leq R_{1}, v\left(\delta_{1}\right) \leq R_{2}, w\left(\delta_{1}\right) \leq R_{3}$ where $R_{1}, R_{2}, R_{3}$ are sufficiently large. Using lemma 1 , system (4.4) and proposition (1.6) from [14], the forward invariance region of system (2.3) is as follows:

$$
\left\{\begin{array}{l}
|u(\tau)| \leq\left|u\left(\delta_{1}\right)\right|+\int_{0}^{\omega}|\dot{u}(\tau)| d \tau<R_{1}+\omega \overline{(\alpha+|\alpha|)}=M_{1} \\
|v(\tau)| \leq\left|v\left(\delta_{1}\right)\right|+\int_{0}^{\omega}|\dot{v}(\tau)| d \tau<R_{2}+\omega \overline{(\xi+|\xi|)}=M_{2} \\
|w(\tau)| \leq\left|w\left(\delta_{1}\right)\right|+\int_{0}^{\omega}|\dot{w}(\tau)| d \tau<R_{3}+\overline{\omega(\mu+|\mu|)}=M_{3}
\end{array}\right.
$$

Observe that the set $K=\left[0, M_{1}\right] \times\left[0, M_{2}\right] \times\left[0, M_{3}\right]$ is forward invariance, compact and convex. Using Brouwer fixed point theorem, see [15], the phase flows $\Phi(\tau)$ of system (2.3) have at least a fixed point say, $\left(u^{*}, v^{*}, w^{*}\right) \in X$ such that $\Phi(\tau) \rightarrow\left(u^{*}, v^{*}, w^{*}\right)$ as $\tau \rightarrow \infty$. By Zorns lemma, and semi-group properties of phase flows $\Phi(\tau)$ of system (2.3), see [16], there exists a maximal element $\mathbb{M}$ satisfying $\left\|\left(u^{*}, v^{*}, w^{*}\right)^{T}\right\|=\left|u^{*}\right|+\left|v^{*}\right|+\left|w^{*}\right|<\mathbb{M}$, where $\mathbb{M}=M_{1}+M_{2}+M_{3}+1$ which is independent of the perturbation parameter $t \in(0,1)$. Taking $\Omega=(u(\tau), v(\tau), w(\tau))^{T} \in X:\|u, v, w\|<\mathbb{M}$; then it is easy to claim that $\Omega$ is an open bounded set in $X$, which verifies lemma 4 (i). When $u(\tau), v(\tau), w(\tau))^{T} \in \partial \Omega \cap \operatorname{Ker} L=\partial \Omega \cap R^{3} ;(u(\tau), v(\tau), w(\tau))^{T}$ is a constant vector in $R^{3}$ with $|u|+|v|+|w|=\mathbb{M}$ and the operator equation $Q N x \neq 0$ which verifies lemma 4(ii). We now verify lemma 4(iii) using lemma (3) as follows. Define a homotopic mapping, say $H(u, v, w ; \lambda)$ : $\operatorname{DomL} \times[0,1] \rightarrow X$ by $H(u, v, w ; \lambda)=\lambda \phi(u, v, w)+(1-\lambda) \psi(u, v, w)$ for $\lambda \in[0,1]$, where

$$
\psi(u, v, w)^{T}=\left\{\begin{array}{l}
\bar{\alpha}-\frac{\overline{1}}{\kappa} \exp u(\tau) \\
\bar{\varepsilon} \frac{\exp u(\tau)}{1+\exp u(\tau)}-\bar{\sigma} \frac{\exp w(\tau)}{1+\exp v(\tau)} \\
\frac{\bar{\beta} \exp v(\tau)}{1+\exp v(\tau)}-\bar{\mu}
\end{array}\right.
$$

Moreover, it can be easily shown that the approximated algebraic system (4.5) has a unique fixed point $\left(u^{*}, v^{*}, w^{*}\right) \in X \subset \mathbb{R}^{3}$ if $\bar{\beta}>\bar{\mu}$. Using homotopy invariance properties of Brouwer's degree, and taking $J=I: \operatorname{Im} Q \rightarrow \operatorname{Ker} L$ then,

$\operatorname{deg}\left(J Q N(\Phi) ; \operatorname{Ker} L \cap \Omega,(0,0,0)^{T}\right)=\operatorname{deg}\left(I Q N(\Phi) ; \operatorname{Ker} L \cap \Omega,(0,0,0)^{T}=\operatorname{deg}\left(\phi(u, v, w)^{T} ; \operatorname{Ker} L \cap\right.\right.$ $\Omega,(0,0,0)^{T}$

$$
=\operatorname{sign}\left|\begin{array}{ccc}
\overline{\bar{\varepsilon}} \exp u(\tau) & 0 & 0 \\
\bar{\varepsilon} \frac{\exp (u(\tau)}{(1+\exp u(\tau))^{2}} & \bar{\sigma} \frac{(\exp w(\tau)}{(1+\exp v(\tau))^{2}} & -\bar{\sigma} \frac{\exp w(\tau)}{1+\exp v(\tau)} \\
0 & \frac{\bar{\beta} \exp v(\tau)}{(1+\exp v(\tau))^{2}} & 0
\end{array}\right|=-1 \neq 0
$$


Therefore, conditions of lemma (4) are satisfied, and system (2.3) has at least one $\omega$ - periodic solution in $D o m L \cap \bar{\Omega}$.

\subsection{Corollary 1}

The set $K=(u(\tau), v(\tau), w(\tau)): 0 \leq u(\tau) \leq M_{1}, 0 \leq v(\tau) \leq M_{2}, 0 \leq w(\tau) \leq M_{3}$ is the absorbing region of phase flows of dynamical system $(2.3)$ in $\Omega$.

\section{$5 \quad$ Uniqueness and Global Attractivity of Periodic Solution}

\subsection{Proposition 2}

Assume the perturbation parameters of dynamical system (2.3) are positive periodic functions, then the dynamical system(2.3) has a unique positive periodic solution, and globally attractive in absorbing region $K$.

Proof: Let $\Phi(\tau)=u(\tau), v(\tau), w(\tau))^{T}$ be a positive periodic solution of of system (2.3) and let $\Psi(\tau)=\left(u^{*}(\tau), y^{*}(\tau), z^{*}(\tau)\right)^{T}$ be any solution of system (2.3) in $K$. We construct a positive definite Lyapunov's functional $F \in C\left[\mathbb{R}^{3} \times \mathbb{R}^{+}, \mathbb{R}^{+}\right]$defined as;

$$
F(\tau)=\left|\operatorname{Inx}(\tau)-\operatorname{In} x^{*}(\tau)\right|+\left|\operatorname{In} y(\tau)-\operatorname{In} y^{*}(\tau)\right|+\left|\operatorname{Inz}(\tau)-\operatorname{In} z^{*}(\tau)\right| .
$$

Using notations in [17] for upper right-derivative of the Lyapunov's functional and differentiating along the direction of trajectories of system (2.3) yields,

$$
\begin{gathered}
D^{+} F(\tau)=\left\{\frac{\dot{x}(\tau)}{x(\tau)}-\frac{\dot{x}^{*}(\tau)}{x^{*}(\tau)}\right\} \operatorname{sign}|x(\tau)-\dot{x}(\tau)|+\left\{\frac{\dot{y}(\tau)}{y(\tau)}-\frac{\dot{y}^{*}(\tau)}{y^{*}(\tau)}\right\} \operatorname{sign}|y(\tau)-\dot{y}(\tau)|+ \\
\left\{\frac{\dot{z}(\tau)}{z(\tau)}-\frac{\dot{z}^{*}(\tau)}{z^{*}(\tau)}\right\} \operatorname{sign}|z(\tau)-\dot{z}(\tau)| \\
\leq \eta_{1}\left|x(\tau)-x^{*}(\tau)\right|+\eta_{2}\left|y(\tau)-y^{*}(\tau)\right|+\eta_{3}\left|z(\tau)-z^{*}(\tau)\right|
\end{gathered}
$$

where

$$
\begin{gathered}
\eta_{1}=\frac{\eta^{m} M_{2}+\beta^{M}}{\left(1+M_{1}^{l}\right)^{2}}-\frac{\alpha^{l}}{\kappa^{M}}>0, \frac{\beta^{m}+\sigma^{m} M_{3}}{\left(1+M_{2}^{l}\right)^{2}}-\frac{\eta^{l}+\eta^{l} M_{1}^{l}}{\left(1+M_{1}\right)^{2}}>0, \\
\eta_{3}=\frac{\sigma^{m}}{\left(1+M_{1}\right)^{2}}-\frac{\left(1+M_{2}\right)^{2}+\sigma^{l} M_{2}^{l}\left(1+M_{1}\right)}{\left(1+M_{1}\right)\left(1+M_{2}\right)^{2}}>0
\end{gathered}
$$

Choose $\delta=\min \left(\eta_{1}, \eta_{2}, \eta_{3}\right)>0$ and integrating both sides yields,

$$
F(\tau) \leq \delta \int_{0}^{\tau}\left(\left|x(\tau)-x^{*}(\tau)\right|+\left|y(\tau)-y^{*}(\tau)\right|+\left|z(\tau)-z^{*}(\tau)\right|\right) d \tau+F(0)<+\infty
$$

The inequality (5.1) guarantees boundedness of the Lyapunov's functional on $[0,+\infty)$, and $(\mid x(\tau)-$ $\left.x^{*}(\tau)|+| y(\tau)-y^{*}(\tau)|+| z(\tau)-z^{*}(\tau) \mid\right) \in L^{1}(0,+\infty)$. Now, applying Barbalat's lemma [18], then $\left(\left|x(\tau)-x^{*}(\tau)\right|+\left|y(\tau)-y^{*}(\tau)\right|+\left|z(\tau)-z^{*}(\tau)\right|\right)$ is uniformly continuous on $[0,+\infty)$ and

$$
\left|x(\tau)-x^{*}(\tau)\right| \rightarrow 0,\left|y(\tau)-y^{*}(\tau)\right| \rightarrow 0,\left|z(\tau)-z^{*}(\tau)\right| \rightarrow 0 \text { as } \tau \rightarrow+\infty .
$$

Therefore, system (2.3) assumed a unique globally attractive positive periodic solution, and trapped in the absorbing region $K$. 


\section{$6 \quad$ Application and Numerical Simulations}

Consider the $\pi$-periodic coefficients of system (2.3) say, $\alpha(\tau)=4.7688+\sin 2 \tau, \kappa(\tau)=2.0064+$ $\sin 2 \tau, \varepsilon(\tau)=1.1249+\sin 2 \tau, \beta(\tau)=0.543+0.2431 \sin 2 \tau, \xi=0.041, \mu=0.3804, \sigma=1.0755, \mu=$ $0.1673, \alpha^{l}=3.7688, \alpha^{m}=5.7688, \beta^{l}=0.2999, \beta^{m}=0.7861, \kappa^{l}=1.0044, \kappa^{m}=3.0064, \varepsilon^{l}=$ $0.1249, \varepsilon^{m}=2.1249, M_{1}^{l}=0.5231, M_{2}^{l}=0.3730, M_{3}^{l}=0.5231, M_{1}=16.9816, M_{2}=17.6788, M_{3}=$ $3.1951, \eta_{1}=0.3602, \eta_{2}=2.2390, \eta_{3}=0.5137, \delta=0.3602$, subject to initial conditions, $x(0)=$ $1.0678, y(0)=1.3730, z(0)=0.6383$. It is easy to examine that the periodic coefficients satisfy boundedness conditions of proposition 1 and 2 .

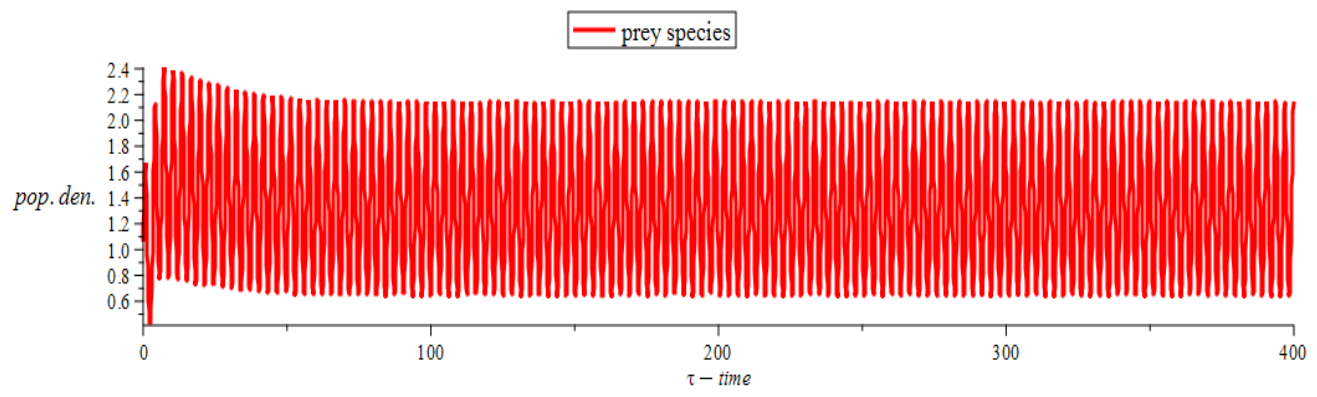

Fig. 1. Globally asymptotically stable periodic solution of prey species of system (2.3) at initial condition $x(0)=1.0678, y(0)=1.3730, z(0)=0.6383$

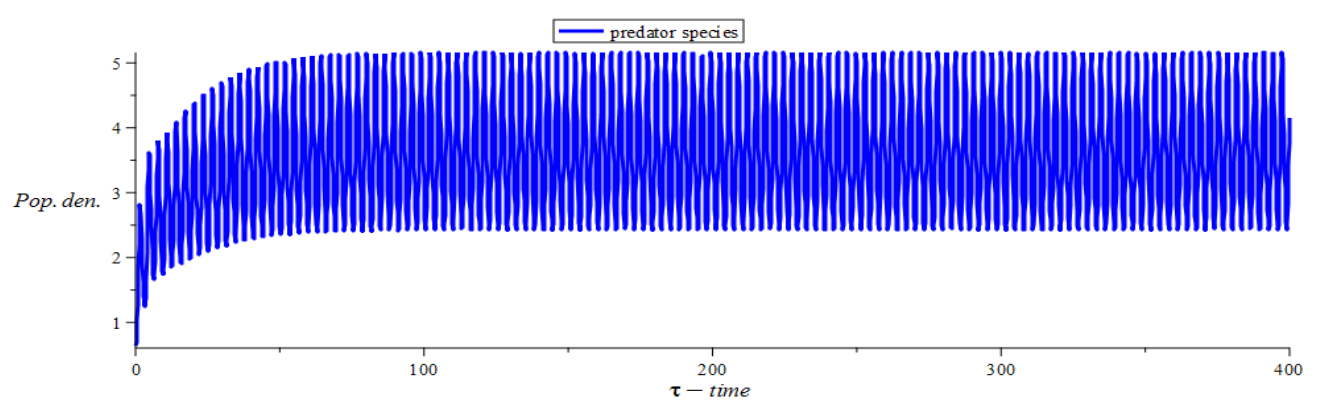

Fig. 2. Globally asymptotically stable periodic solution predator species of system (2.3) at initial condition $x(0)=1.0678, y(0)=1.3730, z(0)=0.6383$

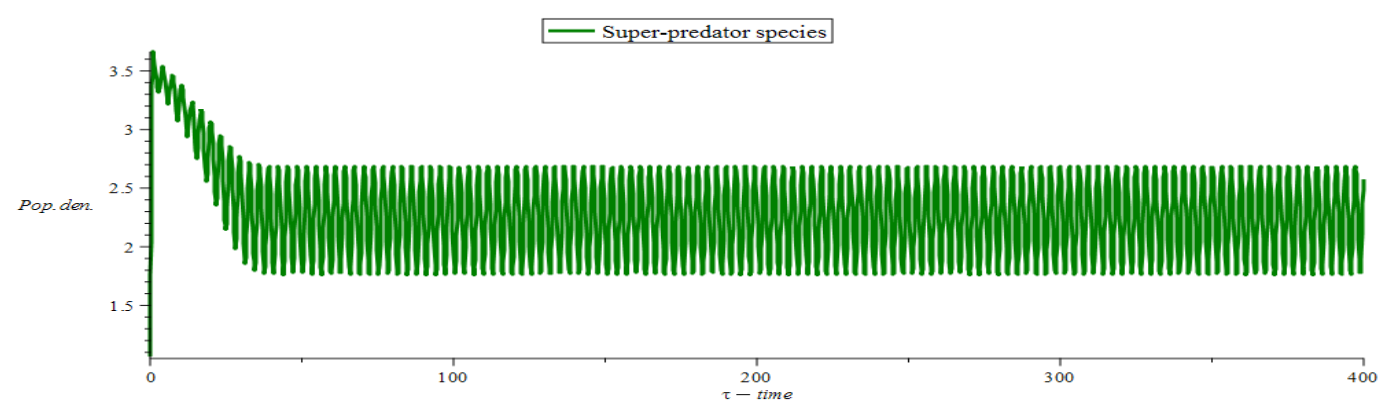

Fig. 3. Globally asymptotically stable periodic solutionsuper-predator species of system (2.3) at initial condition $x(0)=1.0678, y(0)=1.3730, z(0)=0.6383$ 


\section{Conclusion}

This paper has established the necessary conditions for the existence of at least one positive periodic solution of an Extended Rosenzweig-MacArthur tri-trophic food chain model via Brouwers topological degree theory. Also, it has established the sufficient conditions for existence of a unique positive periodic solution of the model using Barbalats lemma and Lyapunovs functional. Consequently, the periodic solution is globally attractive in its invariance region. Thus, this model predicts and depicts a real-life ecological population dynamics as the perturbation parameters assumed periodic oscillations. Its connotes the natural ecological fluctuations.

\section{Acknowledgement}

Author two(2) sincerely appreciates his project supervisor Prof. S. S. Okoya (Chief Editor, Journal of the Nigerian Mathematical Society(NMS); First occupier of Pastor E. A. Adeboye Endowed Professorial Chair in Mathematics University of Lagos, Lagos) for his comments and observations.

\section{Competing Interests}

Authors have declared that no competing interests exist.

\section{References}

[1] Xu C, Li P, Shao Y. Existence and global attractivity of positive periodic solutions for a holling ii two-prey one-predator system. Advances in Difference Equations. 2012;84:1-14.

[2] Xu R, Chaplain MA, Davidson FA. Periodic solutions for a three species lotka-volterra model with time-delays. Mathematical and Computational Modelling. 2004;40:823-837.

[3] Pelen NN, Guvenilir AF, Kaymakcalan B. Necessary and sufficient conditions for existence of periodic solutions of prey-predator dynamic systems with beddignton-deangelis-type functional response. Advances in Difference Equations. 2016;15:1-19.

[4] Zhang Z, Wang Z. Periodic solutions for a two-species non-autonomous competition lotkavolterra patch system with time-delay. Journal of Mathematical Analysis and Applications. 2002;265:38-48.

[5] Agarwal RP, ORegan D. Oscillation and stability of delay models in biology. New York; Springer International Publishing; 2014.

[6] O Regan D, Cho YJ, Chen YQ. Topological degree theory and applications. London; Chapman and Hall CRC, Inc.; 2006.

[7] Curtain RF, Pritchard AJ. Functional analysis in modern applied mathematics. London; Academic Press; 1977.

[8] Gaines RE, Mawhins JL. Coincidence degree and nonlinear differential equations. New York; Springer-Verlag; 1977.

[9] Lynch S. Dynamical systems with applications using maple. New York; Springer Science + Business Media, LCC; 2010.

[10] Shonkwiler RW, Herod J. Mathematical biology. An introduction with maple and MATLAB. New York; Springer Science + Business Media, LLC.; 2009.

[11] Feng W, Freeze M, Lu X. Mathematical analysis of an extended rosenzweig-macarthur model of tri-trophic food chain. Discrete and Continuous Dynamical Systems Series S. 2014;7:1215-1230.

[12] Joshua EE, Akpan ET, Madubueze CE. Hopf-bifurcation limit cycles of an extended rosenzweig-macarthur model. Journal of Mathematics Ressearch. 2016;8(2):22-32.

DOI: $10.5539 /$ jmr.v8n3p22 
[13] Bohner M, Fan M, Zhang J. Existence of periodic solutions in predator-prey and competition dynamics system. Nonlinear Analysis: Real World Applications. 2006;7:1193-1204.

[14] Bohner M, Peterson A. Dynamical equations on time scales: An introduction with applications. USA; Birkhauser; 2003.

[15] Fonseca I, Gangbo W. Degree theory in analysis and applications. New York; Oxford University Press Inc.; 1995.

[16] Saperstone SH. Semi-dynamical systems in infinite dimensional spaces. New York; SpringerVerlag; 1981.

[17] Liao X, Wang L, Yu P. Stability of dynamical systems. Elsevier; 2007.

[18] Gopalsamy K. Stability and Oscillation in Delay Differential Equation of Population Dynamics. Netherlands; Kluwer Academic Publishers; 1992.

(C) 2017 Joshua et al.; This is an Open Access article distributed under the terms of the Creative Commons Attribution License (http://creativecommons.org/licenses/by/4.0), which permits unrestricted use, distribution, and reproduction in any medium, provided the original work is properly cited.

Peer-review history:

The peer review history for this paper can be accessed here (Please copy paste the total link in your browser address bar)

http://sciencedomain.org/review-history/177r2 